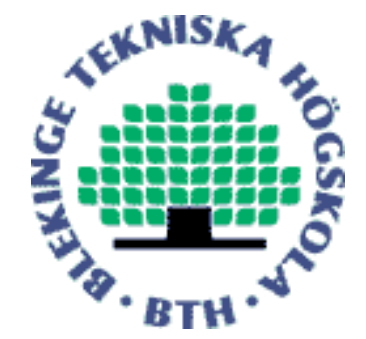

Copyright (C) 2013 IEEE.

Citation for the published paper:

Road travel time prediction - A micro-level sampling approach

Gideon Mbiydzenyuy, Mattias Dahl, Johan Holmgren

16th International Conference on IEEE Intelligent Transport Systems

2013 The Hague

This material is posted here with permission of the IEEE. Such permission of the IEEE does not in any way imply IEEE endorsement of any of BTH's products or services Internal or personal use of this material is permitted. However, permission to reprint/republish this material for advertising or promotional purposes or for creating new collective works for resale or redistribution must be obtained from the IEEE by sending a blank email message to pubs-permissions@iee.org.

By choosing to view this document, you agree to all provisions of the copyright laws protecting it. 


\title{
Road travel time prediction - A micro-level sampling approach
}

\author{
Gideon Mbiydzenyuy ${ }^{1}$, Mattias Dahl ${ }^{2}$, and Johan Holmgren ${ }^{1}$
}

\begin{abstract}
The ability to generate accurate travel time predictions for road freight transport is important when, for example, estimating the arrival times for heavy goods vehicles (HGVs) in order to plan terminal activities. We present a micro-level sampling method for road travel time prediction. The method makes use of historical GPS-data in order to determine the movement of a vehicle from an origin to a destination along a specific route. The method generates a travel time distribution, which can be used to obtain the expected travel time and probabilities for deviations. The method has been illustrated and evaluated in an experiment where the effective travel time was predicted for transport between two terminals. The experiment made use of GPS data that was recorded for two HGVs during a period of two months. An important feature of the method is that it does not need road network information, such as speed limits and number of lanes.
\end{abstract}

\section{INTRODUCTION}

The ability to generate accurate travel time information for road transport is important for many types of transportrelated activities [1]. Travel time information provides decision support for travelers when making route choices; accurate information may help in saving travel time, and improve reliability by avoiding congested sections. In logistics management, the ability to predict travel times, and hence predicting the arrival times, can result in reduced delivery costs.

Nottehed et al. [2] recently presented an interview-based study, which revealed that on-time delivery of freight is an important challenge. The study involved several groups of stakeholders, that is, transport operators, public authorities, universities and networking organizations. They identified the need to reduce uncertainty in the estimation of the arrival time of freight. For some stakeholders, especially in the process industry, on-time delivery of shipments is crucial for efficient planning. In addition, it is important to inform the freight receiver about deviations of the delivery time, so that they are able to take actions and re-plan for another delivery time.

In some situations, it is sufficient to use information about the current traffic situation when making travel time predictions. For example, this is typically the case when predicting the travel time for ongoing short distance transport. Most modern GPS-navigation systems (e.g., $\mathrm{Waze}^{3}$ and TomTom ${ }^{4}$ ) are able to take into account real-time information, such as congestion and accidents, when suggesting routes and

\footnotetext{
${ }^{1}$ School of Computing, Blekinge Institute of Technology, Karlshamn, Sweden

${ }^{2}$ School of Engineering, Blekinge Institute of Technology, Karlskrona, Sweden

${ }^{3} \mathrm{http}: / / \mathrm{www} . w a z e . c o m /$

${ }^{4}$ http://www.tomtom.com/
}

predicting travel times. Most data collection methods involve either site-based or vehicle-based measurements. In site-based measurement, data is collected when vehicles pass specific measurement points in the traffic network [3]. Vehicles are typically identified using techniques, such as automatic number plate recognition, Bluetooth and roadside beacons. In vehicle-based measurements, the vehicles (e.g., probe vehicles) themselves record data [4].

In situations involving future, and in particular, long distance transport, it is not sufficient to make use of travel time estimations based on the current state of the transport network. We argue that such situations require prediction algorithms that are able to generalize historical data into future travel times. In this paper, we present a micro-level method for road travel time prediction, which is based on historical data. The method repeatedly simulates the movement of a vehicle from an origin to a destination along a specific route. It generates a travel time distribution, which can be used to obtain the expected travel time and probabilities for deviations.

An important feature of the proposed method is that it relies solely on historical GPS data recorded under a representative sample of transport conditions. For example, if the forecast indicates there will be snowfall, the input data should be chosen to represent driving in snow conditions. The method does not require any information about static conditions along the road, such as, speed limits and number of lanes.

In Section II, we give an account of related work, followed in Section III by a description of the proposed travel time prediction method. In Section IV, we present an experiment that was conducted in order to evaluate and illustrate the method, and the paper is concluded in Section V.

\section{RELATED WORK}

A lot of research has been conducted in the field of travel time prediction, and several methods have been proposed. Most existing prediction methods are built in a way that they are able to take advantage of the specific type of data that is available. For example, methods that make use of site-based data (e.g., road sensor data) typically focus on trajectory analysis and methods that use vehicle-based data often focus on statistical analysis.

Lee et al [5] suggested a method, which makes use of site-based data, for travel time prediction in an urban road network. The method analyzes the $k$ best paths, which are heuristically selected from a database containing historical travel times for different parts of the network. For each of the links included in the selected paths, the travel time is 
predicted, and the total travel time for each of the $k$ paths is estimated based on the link travel times and potential delays at intersections. Based on a similar idea, Idé and Kato [6] studied the similarities between trajectories in order to predict the travel times for different paths in a road network.

Since site-based data have been shown to be error-prone [7], and due to other, more cost-efficient, possibilities to collect reliable data, vehicle-based methods have gradually started to become an approach of choice. In particular, GPS data has the advantage that large amounts of data can be collected at a reasonable cost. In a recent study, Allström et al. [8] showed the potential of using GPS data for urban travel time estimation. Vanajakshi et al. [9], suggested a method, based on the Kalman filtering technique, where the focus is to predict the effective public bus travel times under heterogeneous traffic conditions. Simroth and Zahle [10] presented a travel time prediction method based on floating car data. In the method, the remaining en-route travel time prediction is performed using a non-parametric distribution-free regression model. Stochastic dependence is assumed between the elapsed time and the remaining time. The remaining time is predicted with the help of partitioning estimates, kernel estimates and $k$-nearest neighbor estimates.

Related work also include methods that combine sitebased (trajectory) and vehicle-based data. Allström et al. [8] combined site-based data (recorded using road-side radar units) and GPS data, for travel time prediction. $\mathrm{Hu}$ et al. [11] suggested two travel time prediction methods: a flowbased and a vehicle-based method. A comparison between the two methods showed that the flow-based method provides significantly more accurate travel time predictions than the vehicle-based method.

As described in this section, there exist travel time prediction methods based on different types of modeling. Common to most of the approaches is that they analyze historical travel patterns related to, for example, origin-destination journey records, previous route choices, and historical travel times, in order to generate a travel time prediction model. It should be mentioned that travel time prediction models might contain errors that are introduced when developing the model, in the form of model bias. In addition, inaccuracies in the available data may negatively influence the results obtained from the model. In this article, we suggest to use the available data directly in the prediction model, and hence we believe that errors that are introduced in the form of bias can be reduced. Even though there is always a risk that the quality of the data that is used is not good enough, we argue that this is a problem with data collection, and not a problem with the actual method. A review of previous research on travel time prediction indicates that micro-level simulation could be used to overcome some of the difficulties of travel time prediction, for example, related to the lack of data or the dependencies between different factors [12]. The proposed travel time prediction method, which is described in the next section, makes use of micro-level simulation, and it uses a set of input GPS data in order to simulate the movement of a vehicle.

\section{A TRAVEL TIME PREDICTION METHOD}

In this section, we describe the proposed road freight travel time prediction method. The method is built according to the idea that the movement of a vehicle is simulated along a specific route that connects an origin and destination for a given transport. The method simulates several journeys along the same route, and thus, it generates a travel time distribution for the considered route. The travel time distribution can be used to obtain the expected travel time and probabilities for deviations.

The method makes use of historical GPS data, which determines the movement of a vehicle from an origin to a destination along a specific route. The route choice can be made, for example, by using optimization software or past experience regarding the performance of different routes connecting the origin and destination. If more than one route is considered interesting to analyze, the method can be applied to each of the routes separately.

At the start of each of the simulated journeys, the simulated vehicle is positioned at the origin of the route under consideration. A historical GPS observation is randomly chosen from the neighborhood of the origin. According to the speed of the selected GPS observation, the vehicle is proceeded along the route for a certain amount of time $\left(t^{s}\right.$ seconds), which gives a new position of the vehicle. From the neighborhood of the new position, another GPS observation is chosen and the vehicle is proceeded for another $t^{s}$ seconds according to the speed of the new GPS observation. This gives yet another vehicle position, and in this way, the vehicle is iteratively proceeded along the route until the destination is reached.

Since the GPS observations that are used to determine the traveling speed of the vehicle are randomly selected, the generated total travel time is, to some extent, stochastic. By simulating several journeys along the same route, according to the principles of Monte Carlo sampling, the method generates a distribution over the predicted travel time.

In Fig. 1., we present the pseudo-code for the proposed travel time prediction method. In the description, we make use of the notation described in TABLE I.

TABLE I

NOTATION USED IN THE METHOD DESCRIPTION.

\begin{tabular}{ll}
\hline$D$ & Destination of the route \\
$g_{k_{m}}$ & The $k_{m}:$ th GPS observation for replication $m$ \\
$G$ & Set of all historical GPS observations \\
$k_{m}$ & Position (iteration) counter for replication $m$ \\
$m$ & Replication (journey) counter \\
$M$ & Number of replications \\
$\mathcal{N}\left(p^{\prime}\right) \subseteq G$ & The neighborhood of a position $p^{\prime}$ \\
$O$ & Origin of the route \\
$p_{k_{m}}$ & The $k_{m}:$ th vehicle position for replication $m$ \\
$t^{s}$ & Simulation time step \\
$t_{m}^{t}$ & Accumulated travel time for replication $m$ \\
$v\left(g^{\prime}\right)$ & The traveling speed for GPS observation $g^{\prime} \in G$ \\
\hline
\end{tabular}




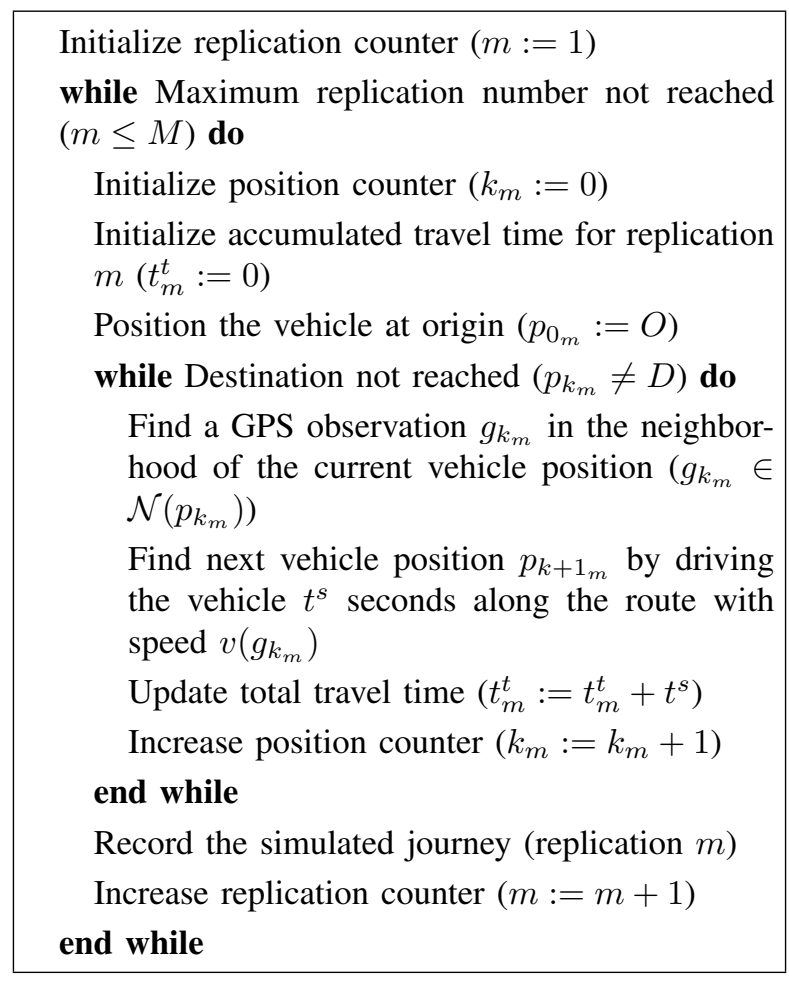

Fig. 1. Pseudo-code for the proposed travel time prediction method.

In the method, it is not specified how the neighborhoods should be defined in order to account for the time and space variability of travel time along a given route. It is assumed that different dynamic factors that lead to time and space variability in travel time can implicitly be accounted for by the choice of neighborhood. Each neighborhood uses GPS data that reflects the conditions of the transport, for example, the level of congestion and weather conditions, that are expected for different parts of the considered route.

One obvious way to specify a neighborhood is to let the neighborhood $\mathcal{N}\left(p^{\prime}\right) \subseteq G$ of a position $p^{\prime}$ consist of all GPS observations that are sufficiently close in distance, that is, $\mathcal{N}\left(p^{\prime}\right)=\left\{g^{\prime}:\left\|g^{\prime}-p^{\prime}\right\|<\delta\right\}$ for some $\delta$. There are also other, more advanced, ways to define the neighborhood of a geographic position, which we exemplify below. Historical GPS data can be used to represent sequences of positions, which specify trips along the route. For several reasons, these historical trips normally do not stretch all the way from the origin to the destination. For example, a vehicle (from which GPS observations are being collected) might temporarily leave the route to visit a terminal. Another way to define the neighborhood is to include only those GPS observations that belong to the same historical trip as the most recently chosen observation. As soon as a historical trip ends, which may occur before the destination is reached, the next neighborhood contains GPS observations that are close enough in distance to the current vehicle position. When using a neighborhood like the one just described, it is possible to "lock" the vehicle to a certain historical trip, which could be good in order to capture infrequently occurring disturbances, which may be represented in the historical GPS data used as input to the method.

In addition, the neighborhood may be chosen in order to reflect certain internal and external conditions that may be specific for the journey. For example, if the weather forecast estimates a $50 \%$ chance of snow for the time of the transport, it is possible to make use of historical data where $50 \%$ of the data represent snow conditions, and $50 \%$ represent other conditions that are typical for the time of transport. If it is known that the vehicle will be heavily loaded for some part of the route, it is possible to only make use of historical GPS data representing heavily loaded vehicles for that particular part of the route. If it is known that the vehicle will travel at night, when the roads are less congested than during daytime, it is possible to make use of only historical GPS data that has been recorded during nighttime.

\section{EXPERIMENTAL EVALUATION}

In order to illustrate and evaluate the proposed travel time prediction method, we have implemented it in Matlab ${ }^{5}$ and conducted an experiment where we studied the movement of an HGV (Heavy Good Vehicle) along a specific route connecting two terminals. When compared with passenger transport, freight transport involves long distances across geo-political boundaries, is highly regulated, and is also influenced by logistic activities such as production and transportation patterns [13]. Even though we considered HGVs in our experiment, it is, however, our belief that the suggested method can be used for prediction of travel time for other types of traffic, depending on which type of data that is used in the method. In the experiment, we analyzed three neighborhoods for determining which GPS observations should be candidates for selecting the speed at which the vehicle should proceed away from a particular geographic position. The experiment involved route selection, data collection, data management, travel time prediction, and output analysis.

\section{A. Description of experiment}

In collaboration with a Swedish transport operator, we selected to study a route, which is operated on a daily basis by two of their HGVs. On-board each of the two vehicles, we installed a GPS-receiver that was used to record data for a period of two months during the winter season of 2012 (February-March). The GPS receivers were configured to record data with a frequency of 10 seconds.

The collected GPS data was imported into Matlab, in order to create a representation of the route, generate trips, and make the simulation runs. We created a representation of the route by selecting positions from the collected set of GPS observations. Observations were selected with a higher density in curves and more sparsely along straight roads in order to obtain a route representation that we considered accurate enough for evaluating and illustrating the suggested method. Between each pair of subsequent positions in the selected set, we added intermediate positions with a distance of approximately 1 meter along the straight lines connecting

\footnotetext{
${ }^{5}$ Matlab is a registered trademark of the Mathworks, Inc.
} 
the two positions. The generated route representation was used when simulating the movement of a vehicle.

From the collected data, we generated trips for each of the vehicles by 1) sorting the collected GPS data in order of time stamp, 2) removing all GPS observations with a distance of more than 10 meters from any point in the route, and 3) traversing the sequence of GPS data recorded for each of the vehicles in order to identify when trips begin and end. Each of the trips begins when it is recognized that the vehicle starts moving after being idle, and it continues until it is recognized that it has a stop for more than six minutes, which we considered large enough to include stops at red lights and occasional queuing, and short enough to detect terminal visits.

In the experiment we evaluated three neighborhoods, each of which was studied in one separate simulation run. In the rest of the paper, we use the terms $N 1, N 2$, and $N 3$ to refer to both neighborhoods and simulation runs.

N1 The neighborhood of a geographical position consists of all GPS observations within a distance of 10 meters.

$N 2$ Identical to $N 1$, except that it includes only GPS observations pointing in the same direction as the considered route.

$N 3$ Identical to $N 2$, except that the neighborhood is restricted to include GPS observations that belong to the same historical trip as the most recently selected GPS observation. For the origin, and if the end of the trip has been reached, the neighborhood is defined as in N2.

In Fig. 2., we illustrate to the left and in the middle, how data was filtered in our experiment. To the right, we exemplify how the vehicle was iteratively proceeded along the route.

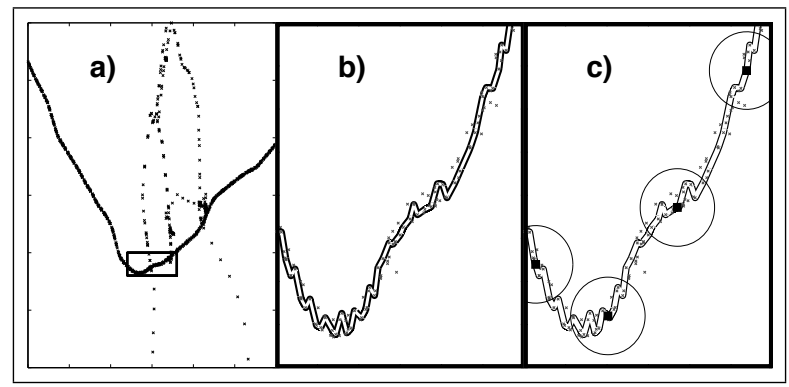

Fig. 2. Data filtering and vehicle movement in the experiment, which include: a) A plethora of GPS observations collected over a period of two months. b) Historical GPS observations along the chosen route are isolated and the representation of the route, to be used in method, is approximated (the two solid lines). c) Transport takes place along the approximated route. The speed of the vehicles are determined by the speed obtained from GPS observations in the neighborhoods (the circles) of the GPS positions for the given vehicle (the boxes in the center of the circles).

For each of the three neighborhoods $(N 1, N 2$, and $N 3)$ we applied the proposed travel time prediction method to simulate 1000 journeys (replications). For each of the simulation runs, we used a simulation time-step $\left(t^{s}\right)$ of 20 seconds.

\section{B. Results and discussion}

As described in Section IV-A the experiment consists of three simulation runs, each of which contains 1000 journeys (or replications)and which corespond to the three neighborhoods ( $N 1, N 2$, and $N 3$ ), shown in Fig. 3. to Fig. 5. We present the obtained travel distributions as histograms together with fitted curves.

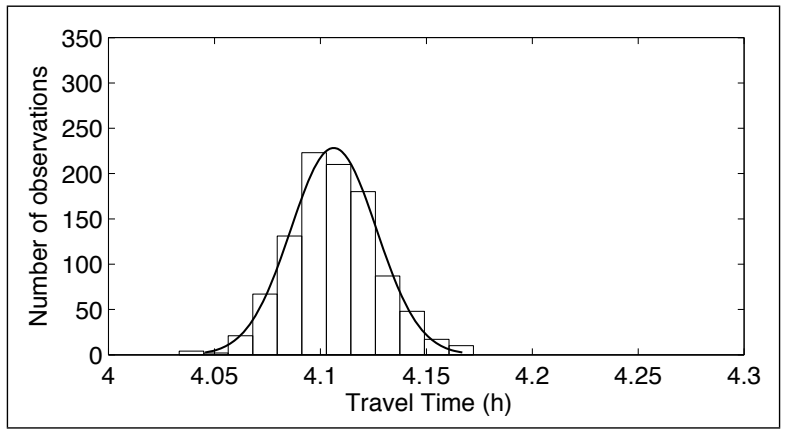

Fig. 3. Histogram for simulation run (neighborhood) $N 1$.

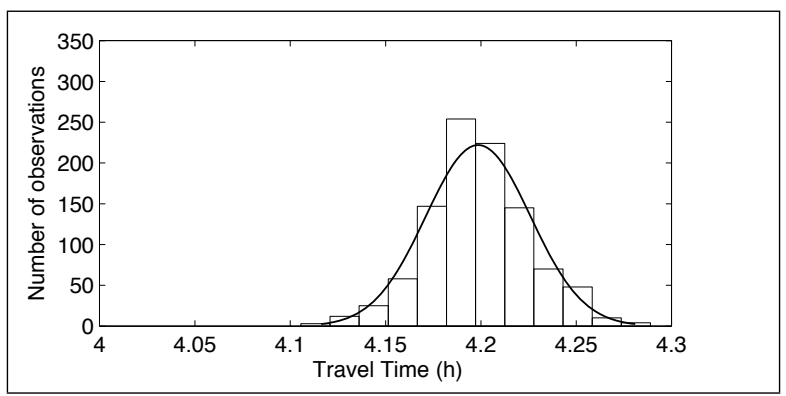

Fig. 4. Histogram for simulation run (neighborhood) $N 2$.

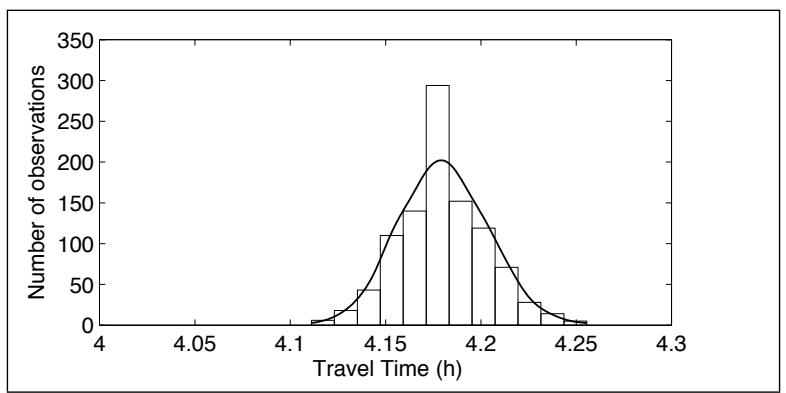

Fig. 5. Histogram for simulation run (neighborhood) $N 3$.

When comparing the mean travel times for the three simulation runs, we observed that $N 1$ gives significantly lower travel times than $N 2$ and $N 3$. The mean travel times were $4.11,4.20$, and 4.18 hours respectively for $N 1, N 2$, and $N 3$. In $N 1$ we considered all recorded GPS observations along the route, regardless of which direction of transport they represent. That is, we used GPS observations representing transport in the direction of the considered route, as well as in the opposite direction. In N2 and N3 we considered GPS observations only in the direction of the route. The mean travel times for $N 2$ and $N 3$ are rather similar, which was expected, since they make use of the same subset of the historical GPS data set. The purpose of introducing neighborhood $N 3$ was to make it possible for the method to capture longer disturbances. As described in Section IVA, this was achieved by restricting the neighborhood of a 
vehicle position to only contain GPS observations belonging to the same trip as the previously chosen GPS observation.

We find it rather reasonable that the observed travel times were longer when considering GPS observations only in the direction of the studied route (in $N 2$ and N3) than when all GPS observations were considered (in N1). The direction in $N 2$ represents transport away from the terminal of the transport operator, and we consider it reasonable to assume that HGVs in most cases are heavier and slower when leaving their home terminal than when returning.

When using neighborhoods such as $N 1$ and $N 2$, in which each GPS observation is chosen randomly without taking into consideration which historical trip the previous GPS observation belongs to, the method will most likely have problems capturing longer disturbances in the travel time prediction. Some historical trips might have characteristics, such as heavy snowfall, that significantly distinguish them from trips that represent normal driving conditions. This possible limitation of $N 1$ and $N 2$ is described using the following example. Let us assume that the movement of a vehicle along a particular route requires a large number of iterations, and that samples are made from, let say, 10 historical trips, of which one is considerably slower than the other trips. In the example, we also assume that each of the trips spans the whole route, and the slower trip is slower all the way from the origin to the destination. In average $10 \%$ of the GPS observations will be chosen from the slower route, and the remaining $90 \%$ of the observations will be chosen from other routes, and this is expected to be the case for each simulated journey even though some disturbances influence transport along the whole route. The expected consequence (in $N 1$ and $N 2$ ) is that the slower route will be partly represented in each of the simulated journeys, instead of being represented to a large extent in a few journeys and not represented at all in most of the journeys. Therefore, we expect neighborhoods such as N1 and $N 2$ to generate results with somewhat lower variance than they should do.

For each of the neighborhoods, we obtained results with rather low variance: $1.21,1.65$, and 1.39 minutes respectively for $N 1, N 2$, and $N 3$. All simulated travel times was observed within intervals of $8.33,11.0$, and 8.57 minutes respectively for $N 1, N 2$, and $N 3$. The main reason for obtaining results with such a low variance is due to that the collected GPS data did not include any major disturbances. For $N 3$ we expected a larger variance than for $N 2$, but in fact, it was smaller. The most plausible reason for this is that neighborhoods such as N3 requires more data, since most of the generated neighborhoods will contain fewer GPS observations compared to neighborhoods like $N 1$ and $N 2$. From the collected GPS data it was possible to generate 26 trips in the direction of interest, and that is probably too small for a neighborhood such as $N 3$.

\section{Problems experienced}

The main threats to the correctness of the experiment concern the collection of data used in the experiment, which in part were caused by the technical limitations of the GPS receivers used in the study; two GlobalSat DG-100 Data Logger ${ }^{6}$.

Each of the GPS receivers has a storage capacity of approximately 43000 GPS observations, and a rechargeable battery that allows for an operation time of approximately 20 hours. The storage capacity limitations made it necessary to copy data and empty the memory at regular intervals during the data collection period. Due to the movement pattern of the vehicles it was possible to visit the terminal of the transport operator once per week in order to copy data from the GPS receivers. In order to make sure that there was always capacity for one week of data collection, we configured the GPS receivers to use a sampling frequency of 10 seconds. In addition, the GPS receivers were configured to $\log$ data only when the traveling speed was at least $5 \mathrm{~km} / \mathrm{h}$.

The battery was continuously charged via the USB connectors in the vehicles, however, we experienced occasionally that the GPS receivers accidentally had (by mistake) been turned off. This caused some gaps in the collected data, since it could take up to a week to realize that a GPS receiver was turned off. In addition, one HGV was on hold for one week in workshop with the GPS receiver stuck in the driver's cabin.

Altogether, we did not manage to obtain the desired amount of data in order to completely evaluate the proposed travel time prediction method. However, we still believe that the collected amount of data was enough in order to indicate the correctness of the method.

\section{CONCLUDING REMARKS AND FUTURE WORK}

We have presented a micro-level sampling method for road travel time prediction. The method makes use of historical GPS data, which determines the movement of a vehicle from an origin to a destination along a specific route. By making use of Monte Carlo sampling, the method generates a travel time distribution, which can be used to obtain the expected travel time and probabilities for deviations. For each journey, the vehicle is iteratively proceeded along the chosen route, until the destination is reached. In each of the iterations, a historical GPS observation is randomly selected from the set of observations that are included in the neighborhood of the current vehicle position. According to the speed of the selected observation, the vehicle is proceeded along the route for a certain amount of time ( $t^{s}$ seconds), which gives a new position of the vehicle.

The method has been illustrated and evaluated in an experiment where we studied transport on a route connecting two terminals. In the experiment which was conducted in collaboration with a Swedish transport operator, we collected and processed GPS data, evaluated three neighborhoods, and analyzed the results. From the analysis of the results for the different neighborhoods, we are able to conclude that the method seems to perform well. Except that neighborhood $N 3$ generated results with lower variance than $N 2$, which

\footnotetext{
${ }^{6}$ www.usglobalsat.com/
} 
probably is due to that $N 3$ requires more input data, the method performs as we expected it to do. We have discussed preliminary results for neighborhood $N 1$ with the transport operator involved in the experiment, who confirmed that the generated results appear to be reasonable for the studied route.

A possible direction for future development of the proposed method is to include planned stops, for example, for loading, unloading, and resting. By also estimating the duration of stops along the journey, it would be possible to more accurately predict the total duration of a journey, not only the effective travel time. Future work also includes further evaluation and analysis of the method. The experiment was based on a rather small amount of GPS data, and it is relevant to conduct experiments with a larger set of historical GPS data, which covers more trips and more dense data. In addition, it is relevant to perform more tests on the existing data set, for example, by studying transport in both directions in the selected route. In order to analyze the behavior of the method, we analyzed three different neighborhoods (i.e., GPS observations) of a vehicle position. It would be interesting to further investigate what influence the choice of neighborhood has on the results, and how the neighborhood can be chosen in order to calibrate the method.

The travel time prediction method proposed in this paper is versatile and flexible. The incidence of, for example, newly arising roadwork, could easily be included in the prediction by introducing weighting functions in the selection of GPS observations that are used to determine the speeds of the vehicle. In addition, the influence of partially outdated information or seasonality can accordingly be suppressed or emphasized. A major advantage of the proposed method in its current form is that it does not need any underlying map information. However, given that map information is available, it would be possible to use the collected data to generate a classifier that can be used to estimate the travel times for the unexplored parts of the road network. Even though the proposed method has been illustrated and evaluated using historical GPS data describing movement of HGVs, it is our belief that the suggested model can be used also for prediction of travel time for other types of traffic, depending on which type of data that is used in the method. Therefore, in the future, we also intend to explore data from different types of transport.

\section{REFERENCES}

[1] H. E. Lin, R. Zito, and M. A. P. Taylor, "A review of travel-time prediction in transport and logistics," in Proceedings of the Eastern Asia Society for Transportation Studies, vol. 5, 2005, pp. 1433-1448.

[2] H. Nottehed, P. Green, B. Sabel, J. Sundberg, U. Janusson, P. Larsson, and J. Löfgren, "Potential and architecture of an Information Broker in the East West Transport Corridor," Project deliverable, East West Transport Corridor 2 (EWTC2), Tech. Rep. Task 3C Report 1, 2010.

[3] P. J. Tarnoff, D. M. Bullock, S. E. Young, J. Wasson, N. Ganig, and J. R. Sturdevant, "Continuing evolution of travel time data information collection and processing," in Transportation Research Board 88th Annual Meeting, no. 09-2030, 2009.

[4] S. M. Turner, W. L. Eisele, R. J. Benz, and D. J. Holdener, "Travel time data collection handbook," Tech. Rep., 1998.

[5] W.-H. Lee, S.-S. Tseng, and S.-H. Tsai, "A knowledge based real-time travel time prediction system for urban network," Expert Systems with Applications, vol. 36, no. 3, pp. 4239-4247, 2009.

[6] T. Idé and S. Kato, "Travel-time prediction using gaussian process regression: A trajectory-based approach," in Proc. of the 9th SIAM international conference on Data Mining (SDM), 2009, pp. 11831194.

[7] R. Li, G. Rose, and M. Sarvi, "Evaluation of speed-based travel time estimation models," Journal of transportation engineering, vol. 132, no. 7, pp. 540-547, 2006.

[8] A. Allström, D. Gundlegård, and C. Rydergren, "Evaluation of travel time estimation based on lwr-v and ctm-v: A case study in stockholm,' in 2012 14th International IEEE Conference on Intelligent Transportation Systems (ITSC 2012), 2012.

[9] L. Vanajakshi, S. Subramanian, and R. Sivanandan, "Travel time prediction under heterogeneous traffic conditions using global positioning system data from buses," Intelligent Transport Systems, IET, vol. 3, no. 1, pp. 1-9, 2009.

[10] A. Simroth and H. Zahle, "Travel time prediction using floating car data applied to logistics planning," Intelligent Transportation Systems, IEEE Transactions on, vol. 12, no. 1, pp. 243-253, 2011.

[11] T.-Y. Hu, C.-C. Tong, T.-Y. Liao, and W.-M. Ho, "Simulationassignment-based travel time prediction model for traffic corridors,' Intelligent Transportation Systems, IEEE Transactions on, vol. 13, no. 3, pp. 1277-1286, 2012.

[12] H.-E. Lin, R. Zito, and M. A. Taylor, "A review of travel-time prediction in transport and logistics," in Proceedings of the Eastern Asia Society for transportation studies, vol. 5, 2005, pp. 1433-1448.

[13] L. Dablanc, "Goods transport in large european cities: Difficult to organize, difficult to modernize," Transportation Research Part A: Policy and Practice, vol. 41, no. 3, pp. 280-285, 2007.

\section{ACKNOWLEDGMENT}

We would like to express our gratitude to the Swedish transport operator who participated in our experiment. We equally wish to thank the project East West Transport Corridor II, for providing funding to carry out this research. Without their support, we would not have been able to conduct this research. 